Thorax (1974), 29, 387.

\title{
A new bronchus clamp
}

\author{
LES L I E J T T E P L E \\ Liverpool Cardio-thoracic Surgical Centre, Broadgreen Hospital, Liverpool
}

Bronchus clamps for use in lung resection have never been entirely satisfactory. The very large number of models available make this plain. When lung resection presents no problems almost any model of bronchus clamp is suitable. In a technically difficult situation all have shortcomings. The basic requirements of a bronchus clamp are:

1. It must be easy to pass with the minimum of dissection of the bronchus, and therefore curved in a way that is least likely to traumatize the surrounding structures.

2. It must compress and grip only a very short length of the bronchus so as to facilitate lung resection when there is very little free bronchus proximal to the tumour.

3. It must be occlusive and be able to grip without slipping when heavy traction is applied to it.
4. It must not cut through or damage the bronchus.

In order to meet these requirements a clamp has been devised with the blades at an angle of $105^{\circ}$ to the shaft and with a gentle curve that enables it to be slipped around the bronchus hugging the front wall and separating it from adjacent tissues. When closed, it flattens the posterior wall against the anterior in a gentle curve. As an added convenience in use, two models are made, one with a right-hand curve and one with a left-hand curve, so that an instrument could be passed in either direction as required.

The use of Potts type teeth on the blades enables a very thin narrow blade to be made that can hold firmly without trauma. It is essential if such a blade is to be effective that the teeth come together with equal pressures along their length

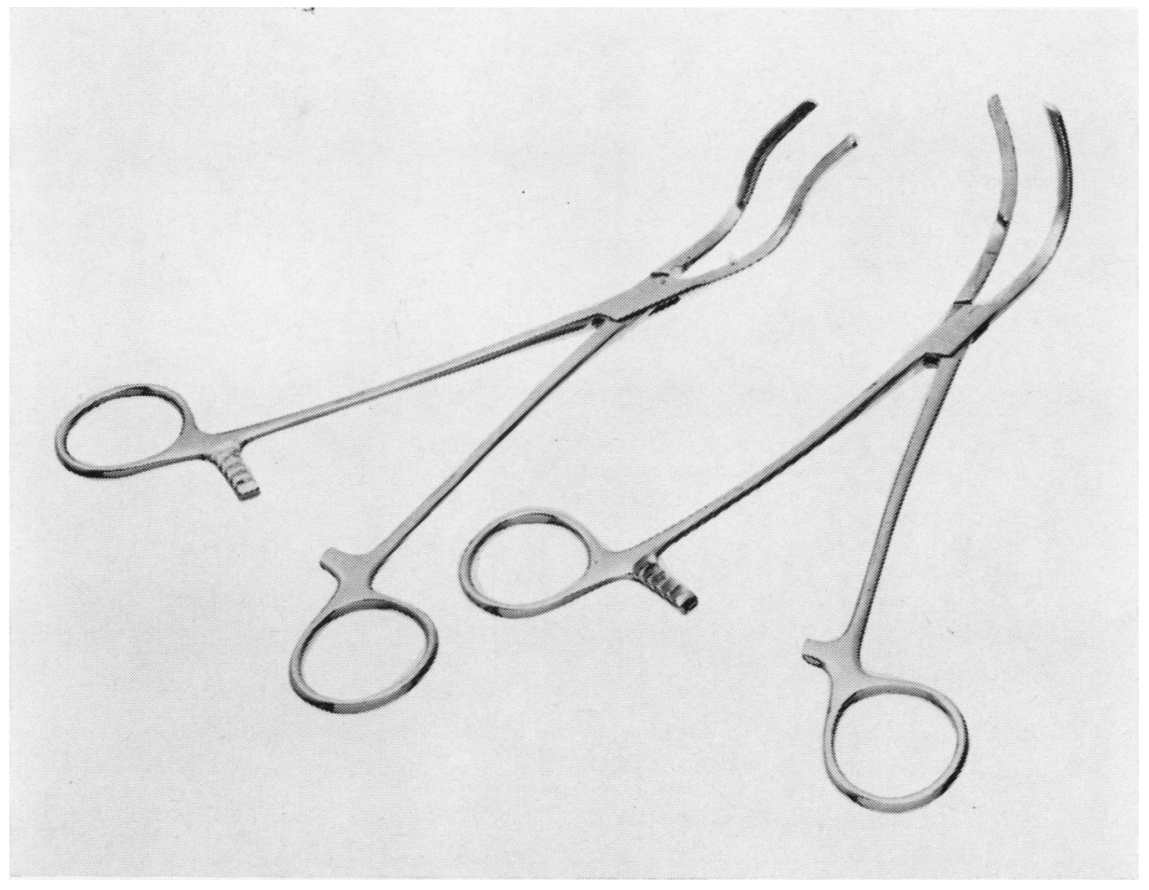


when the angled blades are closed. Since this has been achieved it is possible to grip the bronchus when only 1 or $2 \mathrm{~mm}$ is available and if need be retract the whole lung with it. This enables the surgeon, even with very little space, either to apply a stapling clamp of the type autosuture model TA55 or to close the bronchus with stitches. In practice it proves possible to apply the clamp above a tumour in the main bronchus reaching just up to the carina. A stapling clamp can be applied immediately above it and resection carried out. Placing the clamp tangentially across the side of the trachea enables even more extensive growth to be cleared. Carcinoma of the lung which is of borderline operability becomes very much easier when dealt with in this way.

In the situation in which growth has invaded the left atrium or the pulmonary artery up to the ductus on the left side, it is possible to divide all the other structures, including the bronchus, then lift the lung up by the bronchus clamp so that a vascular clamp can be placed across the bifurca-

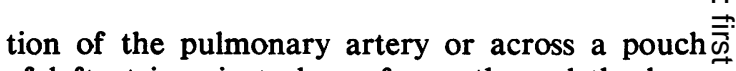
of left atrium just clear of growth, and the lungo cut off above it.

The clamp that has been devised to meet all $\frac{\overline{\bar{c}}}{2}$ these requirements has been continuously in use $\stackrel{\varnothing}{\varnothing}$ for all lung resections for the past six months. There has been no bronchopleural fistula at all in $\overrightarrow{ }$ this time and lung resection has been greatly? facilitated, particularly in the most advanced $\vec{\omega}$ cases. Photographs of the right- and left-handed $O$ models of the clamp demonstrate its structure. It $\vec{x}$ appears to be a very useful and worthwhile ad-ĩ dition to the standard equipment for lung $\dot{\omega}$ resection.

The clamp was manufactured by Down Bros. and음 Mayer \& Phelps Ltd. of Church Path, Mitcham, Surrey, CR4 3UE, and is also available from Downes Surgical (Inc.) Niagara Falls, NY, USA.

Requests for reprints to: Leslie J. Temple, M.B., $\vec{v}$ B.S., F.R.C.S., Liverpool Cardio-thoracic Surgical + Centre, Broadgreen Hospital, Liverpool L14 3LB. 